\title{
The neuroprotective effects of 2-APB in rats with experimentally- -induced severe acute pancreatitis
}

\author{
Karademir $\mathrm{M}^{1}$, Gonul $\mathrm{Y}^{2}$, Simsek $\mathrm{N}^{3}$, Eser $\mathrm{O}^{4}$ \\ Cumhuriyet University School of Medicine, Department of Neurosurgery, Sivas, Turkey. krdmr58@gmail.com
}

\begin{abstract}
AIM: The objective of this study was to determine the neuroprotective effects of 2-aminoethyl diphenyl-borinate (2-APB) on the brains of rats with experimentally-induced severe acute pancreatitis.

MATERIALS AND METHODS: Thirty Spraque-Dawley male rats with an average weight of 200-250 grams were randomly divided into three groups. Group 1: Sham group, Group 2: Severe acute pancreatitis group, Group 3: Treatment group with severe acute pancreatitis, given $2 \mathrm{mg} / \mathrm{kg} 2-A P B$ before pancreatitis onset. In Groups 2 and 3, severe acute pancreatitis was induced by intraperitoneal administration of $1.5 \mathrm{~g} / \mathrm{kg} \mathrm{L}$-arginine with a 1-hour interval. Tumor necrosis factor-a, interleukin 6, pancreatic amylase were all measured. Brain tissue samples were evaluated histopathologically. TUNEL staining method was used to visualize apoptotic cells. RESULTS: In Group 3, it was determined that the density of TUNEL-positive cells in the cerebral cortex has decreased, while the number of Bcl-2-positive cells had increased. In Group 3, it was observed that glial aggregation areas were diminished and histopathological changes were decreased as compared to Group 2. In Group 2, on the other hand, it was observed that in areas with glial cell aggregation, the density of TUNELpositive glial cells had increased, while Bcl-2-positive cell reaction has been feeble.

CONCLUSIONS: It was observed that 2-APB decreases neuronal apoptosis and glial cell aggregation (Tab. 2, Fig. 3, Ref. 21). Text in PDF www.elis.sk.

KEY WORDS: 2-APB, apoptosis, Bcl-2, pancreatic encephalopathy, severe acute pancreatitis.
\end{abstract}

\section{Introduction}

Severe acute pancreatitis (SAP) is an inflammatory disease of the pancreas characterized by a high mortality rate. Acute pancreatitis is caused by activation of pancreatic enzymes that are usually inactive and autolysis causes multi-organ failure via the increased release of inflammatory mediators and the impairment of microcirculation. Pancreatic encephalopathy (PE) is one of the most critical systemic complications that can negatively affect the prognosis of SAP. Although the exact pathogenesis of cerebral injury has not yet been sufficiently illustrated, PE accompanies SAP $18.2 \%$ of the time, with a $67 \%$ mortality rate.

The pathological changes that can occur in the cerebral tissue include impairment of the blood-brain barrier, vasogenic cerebral edema, impairment of microcirculation, ischemia, microhemorrhagic foci, diffuse cerebral demyelination, and apoptosis, especially in neurons $(7,8,10,21)$. Causes of apoptosis include

${ }^{1}$ Cumhuriyet University School of Medicine, Department of Neurosurgery, Sivas, Turkey, ${ }^{2}$ Afyon Kocatepe University School of Medicine, Department of Anatomy, Afyonkarahisar, Turkey, ${ }^{3}$ Balikesir University School of Veterinary Medicine, Department of Histology, Embryology, Balikesir, Turkey, and ${ }^{4}$ Balikesir University School of Medicine, Department of Neurosurgery, Balikesir, Turkey

Address for correspondence: M. Karademir, MD, Cumhuriyet University School of Medicine, Department of Neurosurgery, 58140 Sivas, Turkey. Phone: +90.542.5600117, Fax: +90.346.2191151 a release of cytochrome $\mathrm{c}$ from mitochondria an increase of intracellular $\mathrm{Ca}^{2+}$ and an impairment in the release of anti-apoptotic oncoproteins such as Bcl-2 and Bcl-XL $(1,3,18)$.

Various studies have indicated that the Bcl-2 family executes its anti-apoptotic effect via the regulation of intracellular endoplasmic and mitochondrial calcium metabolism (4). It has been stated that an increase in Bcl-2 expression executes its effect by inhibiting inositol 1,4,5- triphosphate receptors (IP3Rs), decreasing calcium release from plasma membranes and inhibiting the formation of mitochondrial calcium-mediated apoptotic signals (2).

2-APB, which is primarily used as a calcium channel blocker, is a pharmacological agent that has a decreasing effect on IP3Rmediated calcium release by antagonizing inositol 1,4,5 triphosphate receptors. Splettstoesser et al have demonstrated that 2-APB has a decreasing effect on apoptosis by antagonizing IP3R receptors located in plasma membranes and inhibiting the release of intracellular calcium $(14,16)$.

The search for new pharmacological agents that can either inhibit the development of apoptosis or induce an anti-apoptotic effect has escalated substantially; this trend shows promise for producing new treatments for cancer, neurodegenerative diseases after severe acute pancreatitis developed pancreatic encephalopathy and multi-organ failure, and similar pathologies. This study was conducted to investigate whether or not 2-APB has a neuroprotective effect on the brains of rats with experimentallyinduced SAP and PE. 


\section{Materials and methods}

\section{Animals and treatment protocol}

Study protocols and experimental methods were approved by the Afyon Kocatepe University Ethical Committee of Experimental Animals (24.05.2016/49533702-90), and the experiments were performed according to the guidelines of the National Institute of Health (NIH) on animal use. The rats were allowed ad libitum access to food and drank up to the time of the study. All rats were anesthetized under aseptic conditions with an intramuscular injection of ketamine (90 mg/kg, Pfizer Inc., New York) and xylazine (10 mg/kg, Alfasan International, Woerden, Holland). In total, 30 adult male Sprague-Dawley rats weighing 250-300 grams were randomly divided into three groups of 10 rats each:

Group 1 (sham, $\mathrm{n}=10$ ): $1 \mathrm{~mL}$ of $0.09 \%$ saline was administered intraperitoneally (i.p.).

Group 2 (Severe acute pancreatitis (SAP), $\mathrm{n}=10$ ): $1.5 \mathrm{~g} / \mathrm{kg}$ of L-arginine in $0.9 \%$ saline $(1 \mathrm{ml})$ was administered intraperitoneally, in an interval of 1 hour.

Group 3 (Severe acute pancreatitis + 2-APB treatment (SAP+2$\mathrm{APB}), \mathrm{n}=10): 1.5 \mathrm{~g} / \mathrm{kg}$ of L-arginine in $0.9 \%$ saline $(1 \mathrm{~mL}), 1$ hour apart, and $2 \mathrm{mg} / \mathrm{kg}$ 2-APB (Sigma-Aldrich Inc., St. Louis, $\mathrm{MO}$ ) was administered intraperitoneally 10 minutes prior to the first injection of L-arginine.

The test animals were re-anesthetized by ketamine hydrochloride after 24 hours of study, and blood samples were collected from the inferior vena cava for biochemical analysis. The rats were sacrificed at the end of the study by an overdose anesthesia with ketamine/xylazine (90 and $10 \mathrm{mg} / \mathrm{kg}$, respectively; ip).The right and left cerebral lobes were removed for histopathological and immunohistochemical examination.

\section{Biochemical analyses}

Blood samples were kept at room temperature for 2 hours to ensure proper clotting. The samples were then centrifuged at 2500 g at $4{ }^{\circ} \mathrm{C}$ for 15 minutes and stored at $-20^{\circ} \mathrm{C}$ until analysis. Double sandwich enzyme-linked immunosorbent assay kits (eBioscience) were used to measure the serum concentrations of tumor necrosis factor (TNF- $\alpha$ ) and interleukin 6 (IL-6). Serum amylase enzyme activity (U/l) was determined using the Phedebas test.

\section{Histopathological and immunohistochemical analysis}

The brain tissue was fixed with $10 \%$ formalin, dehydrated, and then embedded in paraffin; serial sections were then cut using a microtome. These sections were stained with hematoxylin and eosin for histopathological evaluations. Further immunohistochemical analyses of the cerebral cortex were obtained via staining with TdT-mediated UTP-biotin nick end labeling (TUNEL, S7100, Chemicon International, USA) and Bcl-2 (N-9, Santa Cruz Biotechnology, diluted to 1:200).

For apoptotic analysis, sections with a thickness of 5-6 $\mu \mathrm{m}$ were deparaffinized and rehydrated. To evaluate DNA fragmentation associated with apoptosis, TUNEL was performed using in situ cell death as described by Knoblach et al (11). The sections were fixed in $1 \%$ paraformaldehyde (for 10 minutes) and refixed in precooled ethanol/acetic acid (for 5 minutes at $-20{ }^{\circ} \mathrm{C}$ ). After that, the samples were incubated with $3 \% \mathrm{H}_{2} \mathrm{O}_{2}$ (for 5 minutes), an equilibration buffer (for at least 10 minutes) and then with a working-strength TdT enzyme (for 60 minutes at $37^{\circ} \mathrm{C}$ ), at which point the sections were agitated (for 15 seconds at room temperature). The sections were then incubated with a working-strength stop/wash buffer (for 10 minutes), an anti-digoxigenin conjugate (for 30 minutes) and a 3.3'-diaminobenzidine (DAB) solution.

Bcl-2-positive cells, which protect against apoptosis, were stained via the streptavidin-biotin-peroxidase method. For inhibition of endogenous peroxidase activity, tissue samples were treated with $0.3 \% \mathrm{H}_{2} \mathrm{O}_{2}$ and then incubated in $5 \%$ normal bovine serum to reduce non-specific staining. These sections were then incubated with a primary antibody (Bcl-2: N-9, Santa Cruz Biotechnology), a biotinylated secondary antibody, and a streptavidin-biotin-HRP for 1 hour, and then with DAB substrate kits for 3-5 minutes to obtain immunolabelling. Finally, nuclei were counterstained with Mayer's hematoxylin.

Image analysis was performed using a personal computer, camera, software (Olympus SC30), and an optical microscope. Sections of the tissues were evaluated for histopathological and immunohistochemical examinations. For TUNEL and Bcl-2 immunohistochemical positive cell counting, visuals of the brain cortex were counted in each section under an Olympus CX41 light microscope. These cell numbers were used to measure the balance between cell survival and cell death, and the TUNEL/ Bcl- 2 ratio was calculated by dividing the $\%$ of TUNEL $(+)$ cells by the $\%$ of Bcl-2 (+) (15).

In addition, the intensity of staining with antibodies was subjectively scored as follows: $\mathrm{A}=$ Non-reactivity, $\mathrm{B}=$ Weak, individualized cell reactivity in $\leq 25 \%$ of the cerebral cortex, $\mathrm{C}=$ Mild to moderate reactivity in $\leq 50 \%$ of the cerebral cortex, $\mathrm{D}=$ Strong reactivity in $\leq 75 \%$ of the cerebral cortex, and E = Very strong reactivity in $>75 \%$ of the cerebral cortex [20]. The average staining intensity was calculated by the formula: $[(\mathrm{A} \times 1)+$ $(\mathrm{B} \times 2)+(\mathrm{C} \times 3)+(\mathrm{D} \times 4)+(\mathrm{E} \times 5) /(\mathrm{A}+\mathrm{B}+\mathrm{C}+\mathrm{D}+\mathrm{E})]$, and reported as follows:

i) Not detected $(-)=0.00$,

ii) Rare $(+)=0.01-1.00$,

iii) Small number $(++)=1.01-2.00$,

iv) Moderate amount $(+++)=2.01-3.00$,

v) High number $(++++)=3.01-4.00$

\section{Statistical analysis}

All values were expressed as a mean \pm standard deviation. The numbers of TUNEL and Bcl-2 immunopositive cells were analyzed using a one-way analysis of variance (ANOVA). Significant differences among treatment means were determined using Duncan's test. Differences with a $\mathrm{p}<0.05$ were considered significant.

\section{Results}

\section{Biochemical evaluations}

Amylase levels in the serum were significantly higher in SAP and $\mathrm{SAP}+2-\mathrm{APB}$ groups compared to the control group $(\mathrm{p}<0.05)$. 
Tab. 1. The mean Amylase, TNF- $\alpha$, and IL-6 levels.

\begin{tabular}{ccccc}
\hline Groups & Amylase & TNF- $\alpha$ & IL-6 & \\
\hline 1 & $326.30 \pm 43.14$ & $18.25 \pm 3.16$ & $13.01 \pm 1.14$ & \\
2 & $1345.28 \pm 124.96^{*}$ & $27.86 \pm 1.28^{*}$ & $18.37 \pm 2.15$ & $*$ \\
3 & $1252.60 \pm 165.16^{*}$ & $23.59 \pm 2.56^{*}$ & $16.48 \pm 1.55$ & $*$ \\
\hline
\end{tabular}

1: Sham group, 2: SAP group 3: SAP + 2-APB $(2 \mathrm{mg} / \mathrm{kg})$ treated group

Superscripts * in the same column indicate significant differences $(\mathrm{p}<0.05)$ according to the experimental groups.

Mean values of TNF- $\alpha$ in the serum were significantly higher in the $\mathrm{SAP}$ and $\mathrm{SAP}+2-\mathrm{APB}$ groups than in the control group $(\mathrm{p}<0.05)$. Mean values of IL- 6 in the serum were significantly higher in the pancreatitis group than in the control group $(\mathrm{p}<0.05)$ (Tab. 1).

\section{Histopathological and immunohistochemical evaluations}

In this study, the apoptotic and anti-apoptotic activities of the cerebral cortical neurons were detected by TUNEL and Bcl-2 proteins, with in situ cell death detection kits and streptavidin-biotinperoxidase staining, respectively. Cytoplasmic staining of the cell for Bcl-2 and nuclear staining for TUNEL were determined to be positive in all groups. The results of morphometric and immunohistochemical staining are shown in Table 2.

In Sham group rats, it was observed that molecular-, granular-, pyramidal- and multiform-shaped cellular layers that form the cerebral cortex were in a regular histological structure under the pia
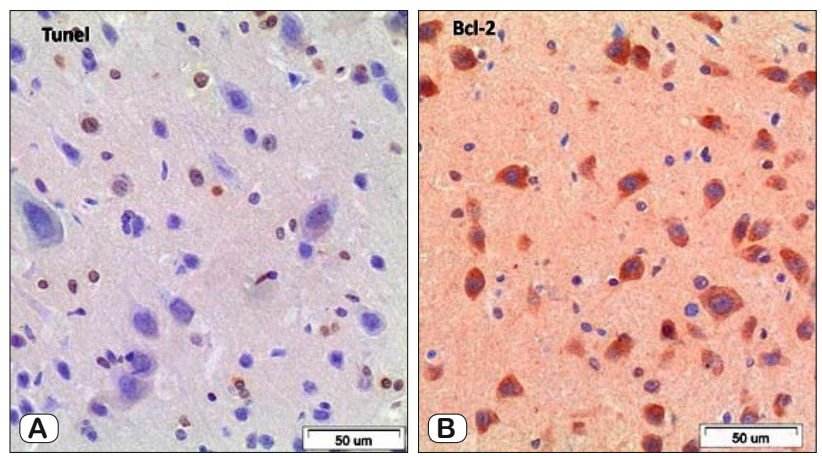

Fig. 1. Control group, arrows: Tunel and Bcl-2 immunopositive cells in the brain.

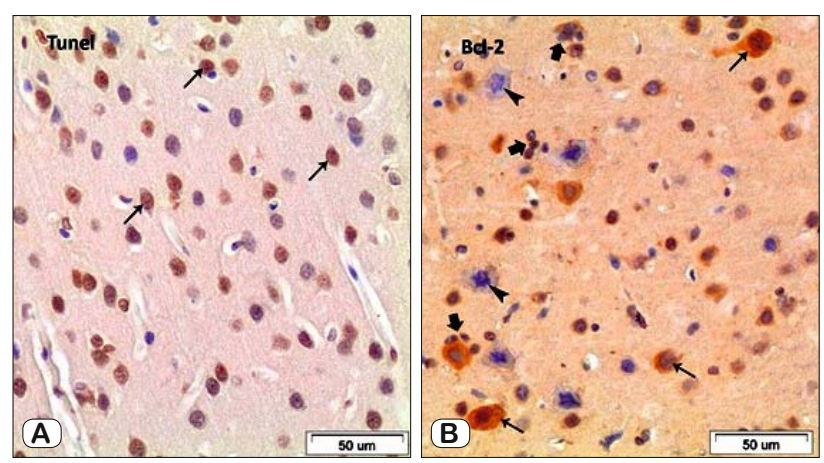

Fig. 2. A-B. Pancreatitis group, arrows: Tunel and Bcl-2 immunopositive cells in the brain, arrowheads: heterochromatic apoptotic neurons, bold arrows: perineuronal glial satellitosis.
Tab. 2. Histochemical, immunohistochemical and morphological examines in the cerebral cortex.

\begin{tabular}{lcccc}
\hline Groups & $\begin{array}{c}\text { Histopathological } \\
\text { changes }\end{array}$ & $\begin{array}{c}\text { Tunel } \\
\text { positivity }\end{array}$ & $\begin{array}{c}\text { Bcl-2 } \\
\text { positivity }\end{array}$ & $\begin{array}{c}\text { Tunel/Bcl-2 } \\
\text { ratio }\end{array}$ \\
\hline 1 & - & + & ++++ & $14 \pm 0.9 / 90 \pm 3.1^{\mathrm{c}}$ \\
2 & +++ & ++++ & + & $72 \pm 2.5 / 53 \pm 1.6^{\mathrm{a}}$ \\
3 & ++ & ++ & +++ & $38 \pm 1.3 / 62 \pm 2.4^{\mathrm{b}}$ \\
\hline
\end{tabular}

1: Sham group, 2: SAP group 3: SAP+ 2-APB $(2 \mathrm{mg} / \mathrm{kg})$ treated group

The scores reported as follows: $-=$ not detected, $+=$ rare, $++=$ small number, +++ $=$ moderate amount, $++++=$ high number. Different superscripts ${ }^{a, b, c}$, in the same column indicate significant differences $(\mathrm{p}<0.05)$ according to the experimental groups.

mater (Fig. 1A-B). In TUNEL staining performed to determine apoptotic cells, it was shown that the nuclei of cortical neurons were not stained, however, nuclei of some neuroglial cells showed a red-brown reaction in control group (Fig. 2A, arrows). In Bcl2 staining performed to determine anti-apoptotic activity, it was demonstrated that almost all of the neurons were Bcl-2-positive (Fig. 2B, arrows).

In the examination of cerebral cortex slices from Group 2 rats with high dose arginine-induced pancreatitis, partial vacuolization in cortical neurons and focal glial cell aggregation and gliosis was observed in some areas, as well as a low amount of perineuronal glial satellitosis. In slices taken from this group, it was determined that the number of TUNEL-positive cells is higher than those observed in the control group (Fig. 3A, arrows), and the number of Bcl-2-positive cells was significantly higher (Fig. 3B, arrows). It was also determined that chromatin in the nuclei of pyramidalshaped neurons have condensed, the nuclei themselves had shrunk, the structure of the cell membrane had degenerated, and the cells seemed to become apoptotic (Fig. 3B, arrowheads).
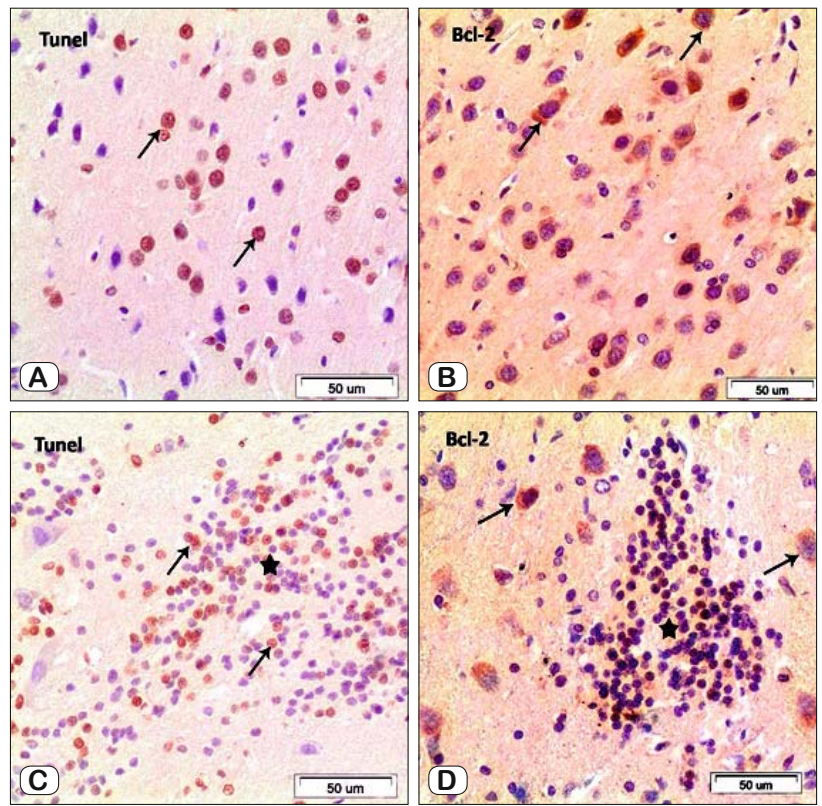

Fig. 3. A-B-C-D. $2 \mathrm{mg} / \mathrm{kg} 2$-APB treated pancreatitis group, arrows: Tunel and Bcl-2 immunopositive cells in brain, star: neuroglia aggregation. 
In the rats given $2 \mathrm{mg} / \mathrm{kg} 2-\mathrm{APB}$ following arginine induction (Group 3), it was determined that glial cell aggregation areas have decreased as compared to Group 2, and histopathological changes had diminished. It was also determined that the density of TUNEL-positive cells have also decreased as compared to Group 2 , while the number of Bcl-2-positive cells had increased. On the other hand, it was determined that the density of TUNEL-positive glial cells have increased in areas with glial cell aggregation, while Bcl-2-positive cell reaction had been feeble (Fig. 3C-3D, arrows).

In addition to the findings described above, histological analyses were also performed. The statistical importance between groups was evaluated by determining the ratio of apoptotic neurons to anti-apoptotic neurons (Tab. 2) $(\mathrm{p}<0.05)$. In the obtained measurements, the TUNEL/Bcl-2 ratio in all groups was found to be 0.15 , $1.36,0.60$, respectively. Based on these results, it appears that treatment with $2 \mathrm{mg} / \mathrm{kg} 2-\mathrm{APB}$ decreases neuronal apoptosis and glial cell aggregation, and increases the synthesis of the protein Bcl-2.

\section{Discussion}

In cases of severe acute pancreatitis, the most critical factor in the development of extra-pancreatic complications is an overactivation of the immune system. An increased release of inflammatory mediators such as TNF- $\alpha$ and IL- 6 causes multi-organ failure (SIRS, or systemic inflammatory response syndrome). Pancreatic encephalopathy, which is observed following SAP, is one of these systemic extra-pancreatic complications, and generally results in mortality; an increase of TNF- $\alpha$ and IL- 6 causes vasogenic cerebral edema by modifying the permeability of the blood-brain barrier. Farkas et al reported that in rats with experimentally-induced severe acute pancreatitis, there is a significant increase in the serum TNF- $\alpha$ and IL- 6 and that this increase causes vasogenic cerebral edema by affecting the blood-brain barrier $(6,7,18)$. Czako et al have stated that in rats in which severe acute pancreatitis was formed by introducing intraperitoneal L-arginine at 12 and 24 hours, there was an apparent increase in the levels of TNF- $\alpha$ and IL-6 (5). In our study, a significant increase in serum values of TNF- $\alpha$ and IL-6 was also observed in rats in which severe acute pancreatitis was induced via intraperitoneal L-arginine, thus supporting the literature. In the 2-APB treatment group, this increase was limited.

Apoptosis, which is defined as programmed cell death, is a process that results in the deformation of cell membranes, breakage in the cell nucleus, chromatin condensation, cell shrinkage, and ultimately cellular suicide resulting from biochemical and histopathological changes associated with morphological degeneration. Whereas apoptosis suppresses the synthesis of anti-apoptotic oncoproteins such as Bcl-2 and Bcl-xl, the synthesis of pro-apoptotic proteins such as Bax and Bak forms the basis for the apoptotic process (9). In experimental SAP and after development of PE, impairment of normal calcium signal pathways is known to be one of the early stages of apoptosis. An extended increase of calcium ion concentration is toxic to central nervous system cells and many cell types, causes mitochondrial dysfunction, degeneration of the cytoskeleton, and degradation of catabolic enzymes (12). This chain of events, caused by impairment in calcium metabo- lism, leads to activation of intracellular apoptotic signal pathways and ultimately, apoptosis. An increase in the permeability of the blood-brain barrier causes apoptosis in and demyelinization of cerebral cells, primarily by increasing intracellular calcium entry. Boehning et al reported that cytochrome $\mathrm{c}$, which is released from mitochondria, amplifies IP3R receptor functions, increases intracellular calcium, and supports the formation of apoptotic signals by binding to IP3R receptors (3). IP3Rs are located in the endoplasmic reticulum, nuclear membranes of almost all cells, and in the plasma membrane of some cell types. Cytosolic $\mathrm{Ca}$ is the primary messenger that regulates IP3R gates (17).

In order to find a new method for preventing the apoptotic process, researchers attentions have been drawn to IP3R, which provides a way to release intracellular calcium and selective calcium channel blockers, and which therefore prevents intracellular calcium entry through the receptors. Ding et al have shown that in rats with PE developed after SAP, the use of nifedipine which has an antagonistic calcium channel effect preserves the semipermeability of the blood-brain barrier by acting as neuroprotective agent (12).

2-Aminoethoxydiphenyl borate (2-APB) is a pharmacological agent that irreversibly inhibits the release of intracellular calcium by blocking inositol 1,4,5 triphosphate-mediated calcium channels. In the literature, the first study concerning 2-APB and its effect on cytosolic calcium release was conducted by Takuyama et al In their study, which involved cerebellar microsomal cells in rats, it was shown that 2-APB inhibits the release of inositol $1,4,5$ triphosphate-mediated intracellular calcium without binding to IP3R, and with the addition of 2-APB to extracellular areas, the cytosolic calcium ratio decreases (13).

Peppiat et al demonstrated that 2-APB decreases not only cytosolic calcium by inhibiting inositol 1,4,5 triphosphate-mediated intracellular calcium channels, but also influences mitochondrial calcium signal pathways by influencing mitochondrial morphology with different intracellular processes (2). Yildar et al reported that 2-APB might reduce the damage of distant organs in rats with L-Arginine induced pancreatitis by inducing calcium homeostasis (19). In our study, we detected the neuroprotective effect of 2-APB, which acts as a calcium channel blocker in the brain by decreasing intracellular calcium entry; we determined this by administering 2 $\mathrm{mg} / \mathrm{kg} 2$-APB to rats that exhibited cerebral cortical cell apoptosis following pancreatic encephalopathy.

The mitochondrial metabolic pathway of apoptosis is regulated by members of the Bcl-2 family, which is composed of both anti-apoptotic and pro-apoptotic members. The Bcl-2 family is the oncoprotein group which plays the most crucial role in the regulation of apoptosis. It has been reported that anti-apoptotic members such as Bcl-2 and Bcl-XL protect cells from many different apoptotic stimuli and thus play a vital role in cell survival. It has also been shown that the Bcl-2 family executes its anti-apoptotic effect via regulation of intracellular endoplasmic and mitochondrial calcium metabolism. Basset et al claimed that an increase in Bcl-2 expression decreases calcium release from plasma membranes by inhibiting inositol 1,4,5- triphosphate receptors (IP3R) and prevents the development of mitochondrial calcium-mediated 
apoptosis $(2,4,18)$. In this study, we determined that in Bcl-2 staining, performed to determine anti-apoptotic activity, almost all of the neurons had Bcl-2-positive cytoplasms. In the examination of cerebral cortical slices of Group 2 rats, which were not treated and in which pancreatitis formed via high dose L-Arginine, it was determined that the number of Bcl-2-positive cells was substantially lower and the number of TUNEL-positive cells was extremely high when compared to the control group. In Group 3, which was treated by applying $2 \mathrm{mg} / \mathrm{kg} 2-\mathrm{APB}$, it was determined that areas of glial cell aggregation were decreased and histopathological changes diminished, as compared to Group 2. Besides, it was determined that the number of Bcl-2-positive cells increases, while the density of TUNEL-positive cells decreased, as compared to Group 2.

The results of histopathological analyses showed that, in the group treated with 2-APB (Group 3), decreases in the number of cerebral cortical apoptotic neurons were observed, while the number of anti-apoptotic neurons increased. It was also observed that treatment with 2-APB has a neuroprotective effect and decreases neuronal apoptosis, glial cell aggregation and increases the synthesis of Bcl-2 proteins which exert an anti-apoptotic effect.

\section{Conclusion}

2-APB blocks the development of mitochondrial membranemediated apoptotic signals by decreasing intracellular calcium release. Although the anti-apoptotic and neuroprotective mechanism of 2-APB has not yet been demonstrated, it may also be a promising agent for developing treatments for systemic complications of severe acute pancreatitis that result in mortality due to apoptotic conditions such as pancreatic encephalopathy. We contend that more detailed research concerning pharmacological agents which can inhibit mitochondria-mediated apoptotic signal pathways via the regulation of intracellular calcium flow is warranted.

\section{References}

1. Atagun G, Eren Z, Gurkanli I. Role of mitochondria in apoptosis. Turk Scient Rev 2014; (2): 49-53.

2. Basset O, Boittin FX, Cognard C, Constantin B, Ruegg UT. Bcl-2 overexpression prevents calcium overload and subsequent apoptosis in dystrophic myotubes. Biochem J 2006; 395 (2): 267-276.

3. Boehning D, Patterson RL, Sedaghat L, Glebova NO, Kurosaki T, Snyder SH. Cytochrome $c$ binds to inositol $(1,4,5)$ trisphosphate receptors, amplifying calcium-dependent apoptosis. Nat Cell Biol 2003; 5 (12): 1051-1061.

4. Bonneau B , Prudent J , Popgeorgiev N , Gillet G. Non-apoptotic roles of Bcl-2 family: The calcium connection. Biochim Biophys Acta 2013; 1833: 1755-1765.

5. Czako L, Takacs T, Varga IS, Hai DQ, Tiszlavicz L, Hegvi P, Mandi Y, Matkovics B, Lonovics J. The pathogenesis of Larginine- induced acute necrotizing pancreatitis: Inflammatory mediators and endogenous cholecystokinin. J Physiol Paris 2000; 94 (1): 43-50.

6. Ding Z, Liu J, Lin R, Hou XH. Experimental pancreatitis results in increased blood-brain barrier permeability in rats: a potential role of mcp1. J Dig Dis 2012; 13 (3): 179-185.
7. Farkas G, Martona J, Naqy Z, Mandi Y, Takacs T, Deli MA, Abraham CS. Experimental acute pancreatitis results in increased blood-brain barrier permeability in the rat: a potential role for tumor necrosis factor and interleukin 6. Neurosci Lett 1998; 242 (3): 147-150.

8. Jingmin O, Xiping Z, Chun W, Ping Y, Qian Y. Study of dexamethasone, baicalin, and octreotide on brain injury of rats with severe acute pancreatitis. Inflamm Res 2012; 61 (3): 265-275.

9. Karavelioglu E, Boyaci MG, Simsek N, Sonmez MA, Koc R, Karademir M, Guven M, Eser O. Selenium protects cerebral cells by cisplatin induced neurotoxicity. Acta Cirúrgica Brasileira 2015; 30 (6): 394-400.

10. Karne S, Gorelick FS. Etiopathogenesis of acute pancreatitis. Surg Clin North Am 1999; 79 (4): 699-710.

11. Knoblach S.M, Alroy DA, Nikolaeva M, Cernak I, Stoica BA, Faden AI. Caspase inhibitor z-DEVD-fmk attenuates calpain and necrotic cell death in vitro and after traumatic brain injury. J Cereb Blood Flow Metab 2004; 24 (10): 1119-1132.

12. Krüger B, Albrecht E, Lerch MM. The role of intracellular calcium signaling in premature protease activation and the onset of pancreatitis. Am J Pathol 2000; 157 (1): 43-50.

13. Maruyama T, Kanaji T, Nakade S, Kanno T, Mikoshibat K. 2-APB, 2-aminoethoxydiphenyl borate, a membrane-penetrable modulator of ins(1,4,5) $\mathrm{P}_{3}$-induced $\mathrm{Ca}^{2+}$ release. J Biochem 1997; 122 (3): 498-505.

14. Peppiatt CM , Collins TJ, Mackenzie L, Conway SJ, Holmes AB, Bootman MD, Berridge MJ, Seo JT, Roderick HL. 2-Aminoethoxydiphenyl borate (2-APB) antagonizes inositol 1,4,5-trisphosphateinduced calcium release, inhibits calcium pumps. Cell Calcium 2003; 34 (1): 97-108.

15. Salakou S, Kardamakis D, Tsamandas AC, Zolota V, Apostolakis E,Tzelepi V, Papathanasopoulos P, Bonikos DS, Papapetropoulos T, Petsas T, Dougenis D. Increased Bax/Bcl-2 ratio up-regulates caspase-3 and increases apoptosis in the thymus of patients with myasthenia gravis. In Vivo, 2007: 21 (1); 123-132.

16. Splettstoesser F, Florea AM, Busselberg D. IP3 receptor antagonist, 2-APB, attenuates cisplatin-induced Ca2-influx in HeLa-S3 cells and prevents activation of calpain and induction of apoptosis. Br J Pharmacol 2007; 151 (8): 1176-1186.

17. Taylor CW, Tovey SC. Ip(3) receptors: toward understanding their activation. Cold spring harb perspect. Biol 2010; 2 (12): a004010.

18. Yazici P, Alizadehshargh S, Akdoğan GG. Apoptosis, regulatory molecules, its relationship with diseases and apoptosis detection methods. Turkiye Klinikleri J Med Sci 2009; 29 (6): 1677-1686.

19. Yildar M, Başbuğ M, Özkan ÖF, Cavdar F, Yaman I, Aksit H et al. The effects of 2-aminoethyl diphenylborinate on L-Arginine induced acute pancreatitis in the rats. Med Sci Discov 2015; 2 (6): 352-357.

20. Yildirim S, Kisa F, Karadeniz A, Yildirim A, Karakoc A, Can I, Kara A, Şimşek N. Effects of pomegranate seed extract on liver paraoxonase and bcl-xL activities in rats treated with cisplatin. J Medic Plant Res 2012; 6 (12): 2317-2323.

21. Zhang XP, Tian H. Pathogenesis of pancreatic encephalopathy in severe acute pancreatitis. Hepatobiliary Pancreat Dis Int 2007; 6 (2): 134-140.

Received August 28, 2018. Accepted October 2, 2018. 